\section{Spectral ANalysis of Random Shrinkage STRESSES IN CONCRETE}

By Zdeněk P. Bažant, ${ }^{1}$ F. ASCE and Tongsheng Wang ${ }^{2}$

ABSTAACT: Random variation of environmental humidity is characterized by its power spectrum, and random variation of pore humidity and stresses in a halfspace is analyzed under the assumption that the problem is linear (or linearized). The diffusion equation and the superposition integral for stress relaxation are used. The dependence of both the creep properties and the drying diffusivity on age is taken into account. Complex variable expressions for the frequency response functions of humidity and stress are obtained and are evaluated numerically. In contrast to nonaging structures, these functions depend on both the current age of concrete and the age when drying starts. The standard deviations of pore humidity and of stress exhibit oscillations about a drifting mean. For typical diffusivities of concrete, the solution is nonstationary for at least $50 \mathrm{yr}$, and for environmental fluctuations whose period does not exceed one year, the fluctuations are not felt at depths over $20 \mathrm{~cm}$ below the surface. Since, even for aging structures, the spectral densities of input and response are related algebraically, the spectral method is computationally more efficient tions of the input and the response are related by integrals.

\section{INTRODUCTION}

Random variation of environmental humidity or temperature has a significant effect on the stresses and deformations in concrete structures The solution is greatly facilitated by considering, as an approximation the material properties to be deterministic and linear. In the linear approximation, the problem is mathematically equivalent to that of random environmental temperature. For the case when the material exhibits no aging and the environmental input is a stationary random process, the response is also a stationary random process. Solution of a typical problem of this type, concerned with random thermal stresses in an infinitely long cylindrical elastic vessel, was pioneered by Heller (13) who used the method of power response spectra. His solution method is, however, inapplicable to concrete because the drying diffusivity and creep strongly depend on age, making the response a nonstationary random process. Random shrinkage stresses in an aging viscoelastic vessel were solved in Ref. 16 by the method of impulse response functions. In this solution, the aging was considered for creep and elastic properties but not for the drying diffusivity of concrete.

The objective of this paper is to solve the problem of a halfspace, and to develop a method of solution that takes into account also the effect of aging on drying diffusivity. The spectral approach is convenient because the environmental humidity variation has a few distinct domi${ }^{1}$ Prof. of Civ. Engrg. and Dir., Center for Concrete and Geomaterials, The Technological Inst., Northwestern Univ., Evanston, IIl. 60201.

${ }^{2}$ Visiting Scholar, Northwestern Univ,; on leave from The Huai River Commission, Bangbu, Anhui, China.

Note.-Discussion open until July 1, 1984. To extend the closing date one month, a written request must be filed with the ASCE Manager of Technical and Professional Publications. The manuscript for this paper was submitted for review and possible publication on January 21, 1983. This paper is part of the Joumal of Engineering Mechanics, Vol. 110, No. 2, February, 1984. (OASCE, ISSN 0733-9399/ 84/0002-0173/\$01.00. Paper No. 18559 . nating periodic components, especially those with periods of $1 \mathrm{yr}$ and 1 day.

\section{Variance and Spectaal Density of Response}

The basic hypothesis of the present solution is that a concrete structure may be treated as a linear system. The input, $f(t)$, of the system is represented by the history of environmental humidity, which may be considered as a stationary random process described by its frequency components. Due to linearity, the effect of each component may be solved separately, and all the responses then superimposed. Consider one input component, $f(t)=A e^{i \omega t}$, of zero mean, frequency $\omega$, and amplitude $A(t=$ time; $A, \omega=$ real numbers; $i=$ imaginary unit). The response is then a certain function, $g\left(x, t, t_{0}\right)=A e^{i \omega t} Y\left(\omega, x, t, t_{0}\right)$, in which $Y$ is a complex function called the frequency response function, $x=$ spatial coordinate for the points in the structure, and $t_{0}=$ age of concrete when the exposure of the structure to input $f(t)$ (environmental humidity) begins. In contrast to nonaging systems (11), the response to a periodic input is nonperiodic since function $Y\left(\omega, x, t, t_{0}\right)$ depends separately on both $t$ and $t_{0}$, and not just on input duration $t-t_{0}$

For a stationary structural response, the statistics of the response are those of the random time variation of the response at a certain point of the structure. This concept, however, is inapplicable if there is aging. One should then imagine an ensemble of many identical structures exposed at the same age, $t_{0}$, to different realizations of the same environment, of the same statistical properties, and then characterize the response by means of statistics of the response values at given age $t$ and given location $x$ over all the structures in the ensemble. This approach, however, would be too tedious for calculations. The random process of the environment, $f(t)$, may usually be considered as stationary, and then the statistics taken over the aforementioned ensemble of all identical structures may be replaced by the statistics of the response $g\left(x, t, t_{0}, \tau\right)$ at fixed location $x$, fixed age $t$, and fixed age at start of exposure, $t_{0}$, as the random environmental history is shifted in time against the instant the structure is cast. Let $\tau$ be the time shift, and $\theta=t+\tau=$ actual time measured, e.g., from the birth of Christ. The statistical characteristics of the response are then those of $g\left(x, t, t_{0}, \tau\right)$ as a function of $\tau(3,4)$.

Consider now a single periodic component of the environment, $f(\theta)=$ $A e^{i \omega \theta}=A e^{i \omega t} \cdot e^{i \omega \tau}$. The response then is $g\left(x, t, t_{0}, \tau\right)=A e^{i \omega t} Y\left(\omega, x, t, t_{0}\right) e^{i \omega \tau}$ The variance of the response may be calculated as the expectation, $E_{\tau}$ of the squared response taken over all values of $\tau$ from $-\infty$ to $\infty$, i.e., of $g\left(x, t, t_{0}, \tau\right) g^{*}\left(x, t, t_{0}, \tau\right)$ in which the asterisk $\left(^{*}\right)$ denotes the complex conjugate. Thus, we have $s_{0 g}^{2}=E_{\tau}\left\{\left[A e^{i \omega t} Y\left(\omega, x, t, t_{0}\right) e^{i \omega \tau}\right]\left[A e^{-i \omega t}\right.\right.$ $\left.\left.Y^{*}\left(\omega, x, t, t_{0}\right) e^{-i \omega \tau}\right]\right\}$.

Noting that $Y Y^{*}=|Y|^{2}$, and that $A=s_{0}=$ standard deviation of the single periodic component of the input, we have

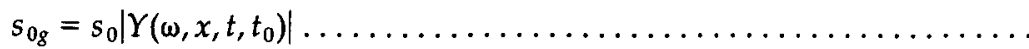

In a preceding work (3), summarized at a recent conference (4), it is shown that, more generally

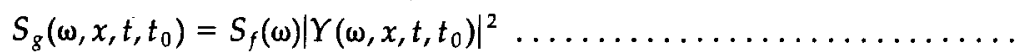


in which $S_{f}$ and $S_{g}$ are the spectral densities of the input and ot the response (3). This relation differs from that for stationary response only by the dependence on $t$ and $t_{0}$. The fact that this relation is algebraic makes the spectral approach computationally more efficient than the impulse response approach, for which the autocorrelation functions of the input and the response are related by integrals. This is especially so if the random input is sufficiently well characterized by only a few periodic components, as is the case for environmental humidity.

In the special case of a single frequency component, $\omega_{0}$, one has $S_{f}(\omega)$ $=2 \pi s_{0}^{2} \delta\left(\omega-\omega_{0}\right)$ and $S_{g}(\omega)=2 \pi s_{0 g}^{2} \delta\left(\omega-\omega_{0}\right)$ in which $\delta\left(\omega-\omega_{0}\right)=$ unit impulse $=$ Dirac delta function. Eq. 2 implies Eq. 1 since $\operatorname{Var}\left[g\left(x, t, t_{0}\right)\right]$ $=\int_{-\infty}^{\infty} S_{g}\left(\omega, x, t, t_{0}\right) d \omega / 2 \pi$ (compare Ref. 3 or 4 ).

\section{Humidity Variation in a Halfspace}

Consider the halfspace $x \geq 0$ whose surface humidity variation is prescribed [Fig. 1( $a, b)]$. The variation of humidity, $h$, inside the halfspace may be assumed to be governed by the linear diffusion equation

$\frac{\partial h}{\partial t}=C(t) \frac{\partial^{2} h}{\partial x^{2}}$

in which $h=$ relative vapor pressure in the pores of concrete; and $C(t)$ $=$ the diffusivity of concrete which is strongly variable with the age, $t$, of concrete, as experimentally demonstrated by Wierig $(17,7)$ and others. Wierig's data may be approximated by

$C(t)=b t^{-m}$.

in which material parameters $b$ and $m$ may be obtained by linear regression of $\log C$ versus $\log t ; m=0.213$; and $b=61.0$ if $t$ is given in days (Fig. 2). To make the solution easier, Eq. 4 is chosen in such a form that
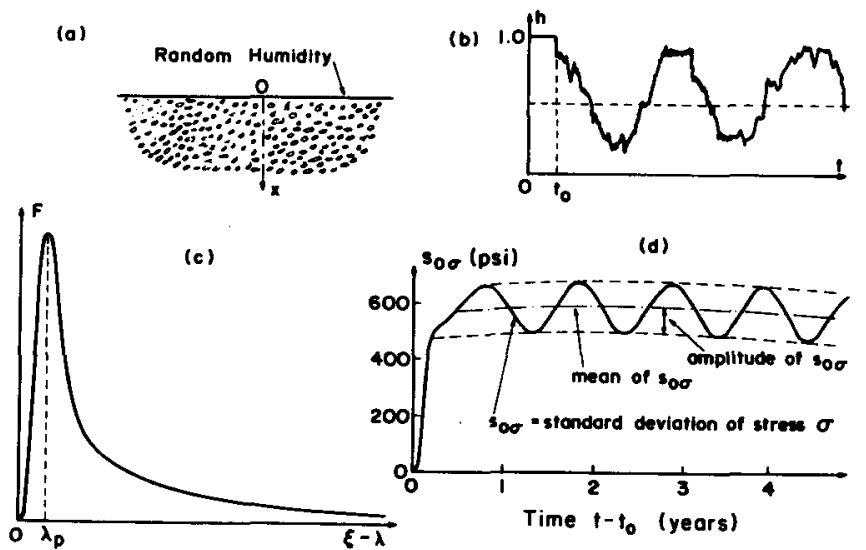

FIG. 1.-(a) Halfspace; (b) Random Variation of Environmental Humidity; (c) Integrand $F(\lambda)$ In Eq. 13; and (d) History of Standard Devlation of Stress

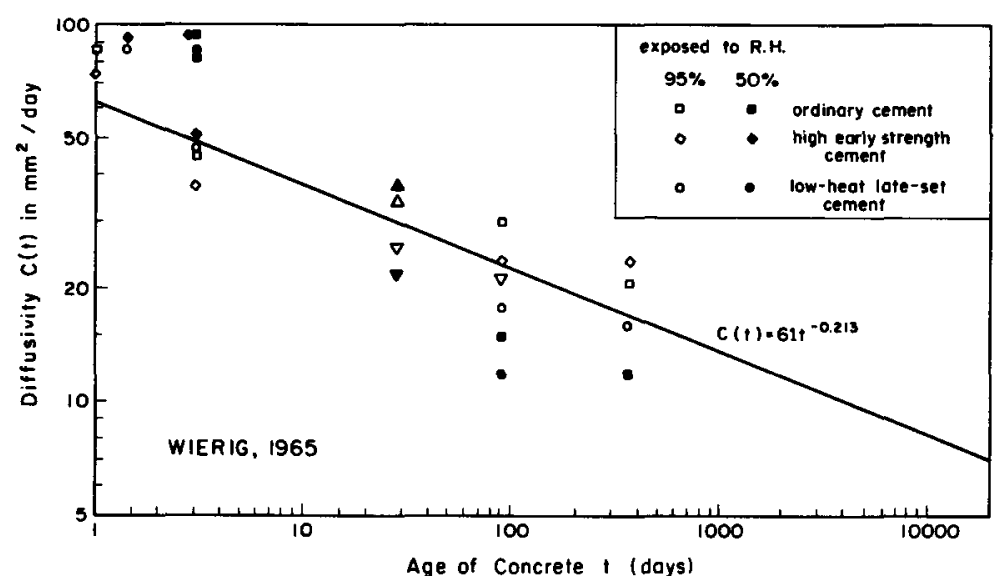

FIG. 2.-Fit of Wlerig's Data (1965) on Ago-Dependence of Diffusivity of Concrete

it can be inverted, i.e., $t=(C / b)^{-1 / m}$. The limit $C \rightarrow \infty$ as $t \rightarrow \infty$, implied by Eq. 4 , is certainly unrealistic, but for typical lifetimes this does not matter.

The variation of humidity is a sum of a deterministic component, $h_{0}(x, t)$, and a randomly fluctuating component of zero mean. The method to solve the first component, which is caused by the difference of the mean environmental humidity and the initial uniform humidity in concrete, is known. Therefore, we consider $h(x, t)$ to be the difference of pore humidity from the mean environmental humidity, and the initial and boundary conditions for solving the fluctuating component of zero mean may then be written as

for $t=t_{0}, \quad x \leq 0: \quad h=0 ;$ for $t \geq t_{0}, \quad x=0: h=e^{i \omega\left(t-t_{0}\right)} \ldots \ldots$ (5)

Here the amplitude of $h$ is assumed as 1.0 since all results are proportional to the amplitude.

Despite aging, an analytical solution is rendered possible by substituting the variable

$\xi=\phi(t)=\int C(t) d t$

which may be called the reduced time. Assuming that $\xi=0$ when $t=$ $t_{0}$, Eq. 6 becomes

$\xi=\phi(t)=\int b t^{-m} d t=\frac{b}{n} t^{n}+C$

in which $C_{0}=\frac{b}{n} t_{0}^{n}, \quad n=1-m$

The inverse of Eq. 7 is

$t=\psi(\xi)=\left(t_{0}^{n}+\frac{n}{b} \xi\right)^{1 / n}$ 
Noting that $\partial h / \partial t=(\partial h / \partial \xi) \partial \xi / \partial t=C(t) \partial h / \partial \xi$, the field equation (Eq. 3) becomes

$\frac{\partial h}{\partial \xi}=\frac{\partial^{2} h}{\partial x^{2}}$

and the boundary conditions (Eq. 5) become

for $\xi=0, \quad x \geq 0: \quad h=0 ;$ for $\xi \geq 0, \quad x=0: \quad h=e^{i \omega\left[\psi(\xi)-t_{0}\right]} \ldots .$. (11)

We see that the transformation of time reduces the problem to a diffusion problem with a constant diffusivity, equal to 1.0 . According to $\mathrm{Du}$ hamel's theorem (10), the solution of this problem is

$h(x, t)=e^{i \omega t} Y_{h}\left(\omega, x, t, t_{0}\right)$

$Y_{h}\left(\omega, x, t, t_{0}\right)=\frac{x}{2 \sqrt{\pi}} e^{-i \omega t_{0}} \int_{0}^{\xi} e^{-x^{2} / 4(\xi-\lambda)}(\xi-\lambda)^{-3 / 2} d \lambda, \quad \xi=\phi(t) \ldots$

which can be verified by substitution into Eqs. 10 and 11 . The actual humidity is the real part of $h(x, t)$, and function $Y_{h}$ is the frequency response of humidity.

For a general random input of given spectral density, the frequency response function given by Eq. 13 makes it possible to determine all the statistical parameters of the response. In particular, according to Eq. 2, the standard deviation of humidity is

$s_{0 h}(x, t)=s_{0}|h(x, t)|=s_{0}\left|Y\left(\omega, x, t, t_{0}\right)\right|$

\section{Variation of Stress in Halfspace}

Changes of pore humidity produce local shrinkage strains, $\epsilon_{s h}(x, t)$ Although the dependence of $\epsilon_{s h}$ on $h$ is nonlinear, it needs to be linearized to make an analytical solution feasible. A linearization is acceptable for humidities between 0.5 and 1.0, and may be written as

$\epsilon_{\text {sh }}(x, t)=\kappa_{\text {sh }} h(x, t)$

in which $\kappa_{s h}$ is a shrinkage coefficient (a real number), which depends on age $t$. This dependence has, however, been neglected in numerical calculations, for lack of experimental data, although the same type of solution is possible even when $\kappa_{s h}$ depends on $t$.

Since the shrinkage strains obtained from Eq. 15 are not compatible, shrinkage stresses are produced. They may be determined from the conditions that the normal stress, $\sigma_{x}$, and the normal strains, $\epsilon_{y}$ and $\epsilon_{z}$ vanish. For the case of an elastic halfspace, in which the shrinkage strains do not vary in the directions parallel to the surface, these conditions would yield the shrinkage stresses

$\sigma_{y}=-\frac{E_{c}}{1-v} \kappa_{s h} h(x, t)$

in which $E_{c}=$ Young's modulus of concrete; and $v=$ its Poisson ratio $\left(\sigma_{x}=0, \sigma_{z}=\sigma_{y}\right)$.
The shrinkage stresses are greatly reduced by creep. Using the elasticviscoelastic analogy, the solution for creep may be obtained simply by replacing $E_{c}$ with the relaxation operator $(1,2)$; this yields the shrinkage stress

$\sigma_{y}(x, t)=-\frac{E}{1-\underline{v}} \kappa_{s h}(t) h(x, t)$

$=-\int_{t_{0}}^{t} \frac{R\left(t, t^{\prime}\right)}{1-v\left(t, t^{\prime}\right)} \kappa_{s h}\left(t^{\prime}\right) \frac{\partial}{\partial t}\left[H\left(t^{\prime}-t_{0}\right) h\left(x, t^{\prime}\right)\right] d t^{\prime}$.

in which $H=$ the Heaviside step function; $E=$ relaxation operator of concrete; $\underline{v}=$ operator corresponding to $v ; R\left(t, t^{\prime}\right)=$ relaxation function of concrete $=$ uniaxial stress at age $t$ caused by a unit normal strain imposed at age $t^{\prime}$; and $\nu\left(t, t^{\prime}\right)=$ associated Poisson ratio function. As a good approximation, $v\left(t, t^{\prime}\right)=v=$ constant. According to Eq. 12, for $t>t_{0}$

$\frac{\partial h\left(x, t^{\prime}\right)}{\partial t^{\prime}} d t^{\prime}=\frac{\partial}{\partial t^{\prime}}\left[e^{i \omega t^{\prime}} Y_{h}\left(\omega, x, t^{\prime}, t_{0}\right)\right] d t^{\prime}$

$=e^{i \omega t}\left[i \omega Y_{h}\left(\omega, x, t^{\prime}, t_{0}\right)+\frac{\partial Y_{h}\left(\omega, x, t^{\prime}, t_{0}\right)}{\partial t^{\prime}}\right] d t^{\prime}$

Substituting this into Eq. 17, and setting

$\sigma_{y}(x, t)=e^{i \omega t} Y_{s}\left(\omega, x, t, t_{0}\right)$

we obtain the following expression for the frequency response of shrinkage stress:

$Y_{s}\left(\omega, x, t, t_{0}\right)=-\int_{t_{0}}^{t} \frac{R\left(t, t^{\prime}\right) \kappa_{s h}\left(t^{\prime}\right)}{1-v\left(t, t^{\prime}\right)} \frac{\partial}{\partial t^{\prime}}\left[e^{-i \omega\left(t-t^{\prime}\right)} Y_{h}\left(\omega, x, t^{\prime}, t_{0}\right)\right] d t^{\prime}$

$-\frac{R\left(t, t_{0}\right) \kappa_{s h}\left(t_{0}\right)}{1-v\left(t, t_{0}\right)} e^{i \omega t_{0}} Y_{h}\left(\omega, x, t^{\prime}, t_{0}\right)$

Here the last term vanishes for all $x$ except $x=0$ because only pore humidity at surface can change instantaneously. The frequency response function in Eq. 20 (which is complex-valued) makes it possible to determine all the statistical characteristics of the response. In particular, the standard deviation of the stress is

$s_{0 \sigma}(x, t)=s_{0}\left|s_{y}(x, t)\right|=s_{0}\left|Y_{s}\left(\omega, x, t, t_{0}\right)\right|$.

As a special case, consider the points on the surface of the halfspace, $x=0$. Evaluating the limit of Eq. 13 for $x \rightarrow 0$, one can verify that $Y_{h}\left(\omega, 0, t, t_{0}\right)=H\left(t-t_{0}\right)=$ Heaviside step function $=1$ if $t>t_{0}$. Eq. 20 then yields

$Y_{s}\left(\omega, 0, t, t_{0}\right)=-\frac{R\left(t, t_{0}\right) \kappa_{s}\left(t_{0}\right)}{1-v\left(t, t_{0}\right)} e^{-i \omega\left(t-t_{0}\right)}$

$-i \omega \int_{t_{0}^{+}}^{t} \frac{R\left(t, t^{\prime}\right) \boldsymbol{\kappa}_{s h}\left(t^{\prime}\right)}{1-\nu\left(t, t^{\prime}\right)} e^{-i \omega\left(t-t^{\prime}\right)} d t^{\prime}$ 
The basic premise of the present solution is complete linearity of the problem, including the diffusion equation, the shrinkage-humidity relation, and the stress-strain relation.

The present method of solution can be applied, in principle, for any structure for which an elastic solution, either analytical or numerical, is available. Note that the method holds even when $R\left(t, t^{\prime}\right)$ depends also on spatial coordinate $x$ (layered halfspace). This is because the conditions $\sigma_{x}=\epsilon_{y}=\epsilon_{z}=0$ remain. However, Eq. 13 per se does not apply if diffusivity $C(t)$ depends on $x$.

It may be also noticed that an analogous solution is possible for thermal stresses. Random environmental humidity history is then replaced by random environmental temperature history, the diffusion equation for pore humidity by the heat conduction equation, and the shrinkagehumidity relation by the relation of thermal expansion to temperature. To apply the present solution to random thermal stresses is simply a matter of changing some notations. In practice, thermal stresses and shrinkage stresses occur simultaneously, but the analysis of combined random shrinkage and thermal stresses is just a matter of superposition of solutions. This is true, however, only as long as both the shrinkage and thermal stress problems remain linear and uncoupled. In practice, these assumptions might be often unrealistic. For example, the thermal and shrinkage problems become coupled if moisture in the surface layer of concrete freezes. Undoubtedly, this would be much more difficult to handle.

\section{Numerical Solution and Examples}

The frequency response functions may be calculated numerically if the time interval, $\left(t_{0}, t\right)$, is subdivided by discrete times $t_{0}, t_{1}, t_{2}, \ldots t_{n}$. Eq. 20 for $x>0$ may then be approximated by the sum

$Y_{s}\left(\omega, x, t_{n}, t_{0}\right)=-\sum_{i=1}^{n} \frac{R\left(t_{n}, t_{i}\right) \kappa_{s h}\left(t_{i}\right)}{1-v\left(t_{n}, t_{i}\right)} e^{-i \omega\left(t_{n}-t_{i}\right)}\left[Y_{h}\left(\omega, x, t_{i}, t_{0}\right)\right.$

$\left.-e^{-i \omega \Delta t_{i}} Y_{k}\left(\omega, x, t_{i-1}, t_{0}\right)\right]$.

For a numerical example, we consider the relaxation function obtained for Ross Dam concrete, which is given in Ref. 8 in the form of Dirichlet series

$R\left(t, t^{\prime}\right)=\sum_{\mu=1}^{m} E_{\mu}\left(t^{\prime}\right) e^{-\left(t-t^{\prime}\right) / \tau_{\mu}}+E_{\infty}\left(t^{\prime}\right) \ldots \ldots \ldots \ldots \ldots \ldots \ldots \ldots \ldots \ldots \ldots \ldots \ldots$

in which $\tau_{\mu}$ are the relaxation times; and $E_{\mu}\left(t^{\prime}\right), E_{\infty}\left(t^{\prime}\right)$ are the Maxwell chain moduli, as specified in Ref. 8. Eq. 24 gives a good approximation within the time range $0.5 \tau_{1} \leq t-t^{\prime} \leq \tau_{\mu}$. In all numerical calculations, the creep Poisson ratio is considered as constant, $v\left(t, t^{\prime}\right)=v=0.18$. The drying diffusivity is $C(t)=61 t^{-0.213} \mathrm{~mm}^{2} /$ day (Fig. 2). The shrinkage coefficient is considered age-independent, and the value $\kappa_{s h}=0.0008$ typical for structural concretes [but too high for dam concretes (18)], is assumed. Comparing the numerical examples from Ref. 16 , note that $\kappa_{s h}$ was considered twice as large there.
In Eq. 24, the relaxation function is considered to be independent of $h(x, t)$ as well as of $\partial h(x, t) / d t$ and the history of $h(x, t)$. In reality, $R\left(t, t^{\prime}\right)$ $h(x, t)$, as well as of $\partial h(x, t) / d t$ and the a linear solution would become impossible. The effect of drying on the a linear solution would becom into account, at creep or relaxation properties could, how least approximately, in the mean sense. For this purpose one would need to subdivide the halfspace into layers and take a different relaxation function, $R\left(t, t^{\prime}\right)$, for the overall drying regime of that layer, in a similar manner as that used in Ref. 9.

A computer program to implement the foregoing solution was written, in complex arithmetic. Numerical evaluation of the integral in Eq. $\exp \left[-(\xi-\lambda) x^{2} / 4\right]$, has a sharp peak at $\lambda=\lambda_{p}=\xi-6 / x^{2}$ [see Fig. 1(c)]. The smaller the value of $x$, the sharper the peak. To integrate accurately, the integration interval $0 \leq \lambda \leq \xi$ was subdivided into subintervals such as $\left(0, \lambda_{p}\right),\left(\lambda_{p}, 2 \lambda_{p}\right)$, followed by double-size subintervals until $\xi$. Within each subinterval, a 16-point Gauss integration was used. Convergence was also tested numerically.

Compared to standard concrete creep problems for steady loads, the Compared to standard concrete creep problems for steady loads, the
amount of computation is here greatly increased by the oscillatory nature of the solution. Unlike those problems, here it is not possible to keep increasine the time steps because, in order to maintain sufficient accuracy, the e step $\left(t_{i}-t_{i-1}\right)$ cannot exceed about $1 / 16$ of the oscillation period, $2 \pi / \omega$. Thus, the required number of time steps is at cillation steps may be needed if a good accuracy is desired for points of small $x$ immediately after the exposure time, $t_{0}$. Then, a finer subdivision is required for small $t-t_{0}$, with time steps increasing in a geometric progression as long as they do not exceed $2 \pi / 16 \omega$.

For the sake of simplicity, calculation results are reported here only for the case of a single periodic component in the input, having the period of one year, $h=s_{0} e^{i \omega\left(t-t_{0}\right)}=0.2 e^{i\left[2 \pi\left(t-t_{0}\right) / 365\right]}$, i.e., its standard deperiod of one year, $h=s_{0} e^{i \omega\left(t-t_{0}\right.}=0.2 e^{i(n)}$ is $\omega=2 \pi / 365$. In Figs. 3 viation is $s_{0}=0.2$ and its circular frequency is $\omega=2 \pi / 365$. In Figs. 35 $3,28,365$ days. Fig. 3(a) shows the histories of humidity (its real part) for points at various depths, $x=2 \mathrm{~cm}, 5 \mathrm{~cm}, 10 \mathrm{~cm}$, and $20 \mathrm{~cm}$. The for points at various depths, $x=2 \mathrm{~cm}, 5 \mathrm{~cm}, 10 \mathrm{~cm}$, and $20 \mathrm{~cm}$. The the case of constant diffusivity. The decrease of diffusivity with age causes the amplitude to decay more rapidly than for constant diffusivity. The humidity oscillations are imperceptible for $x>20 \mathrm{~cm}$. Multi-year environmental periods would influence a greater depth, while periods of less than 1 yr would influence a smaller depth; also, a higher diffusivity would cause the oscillations to reach deeper.

Fig. 4 shows the time variation of the standard deviation of humidity, $s_{0 h}$. The greater the depth, the smaller the value of $s_{0 h}$. It is noteworthy that $s_{0 h}$ oscillates, too. These oscillations decay with time and become graphically undiscernible at $t-t_{0}=10 \mathrm{yr}$, after which the plot of $s_{0 h}$ is grantially a downward inclined straight line in the $\log \left(t-t_{0}\right)$ scale. (Actually, even for constant diffusivity, i.e., nonaging material, $s_{0 h}$ os(Actually, even for to initial condition for a limited period of time until a stationary response is reached.) Generally, the standard deviation, $s_{0 h}$, 

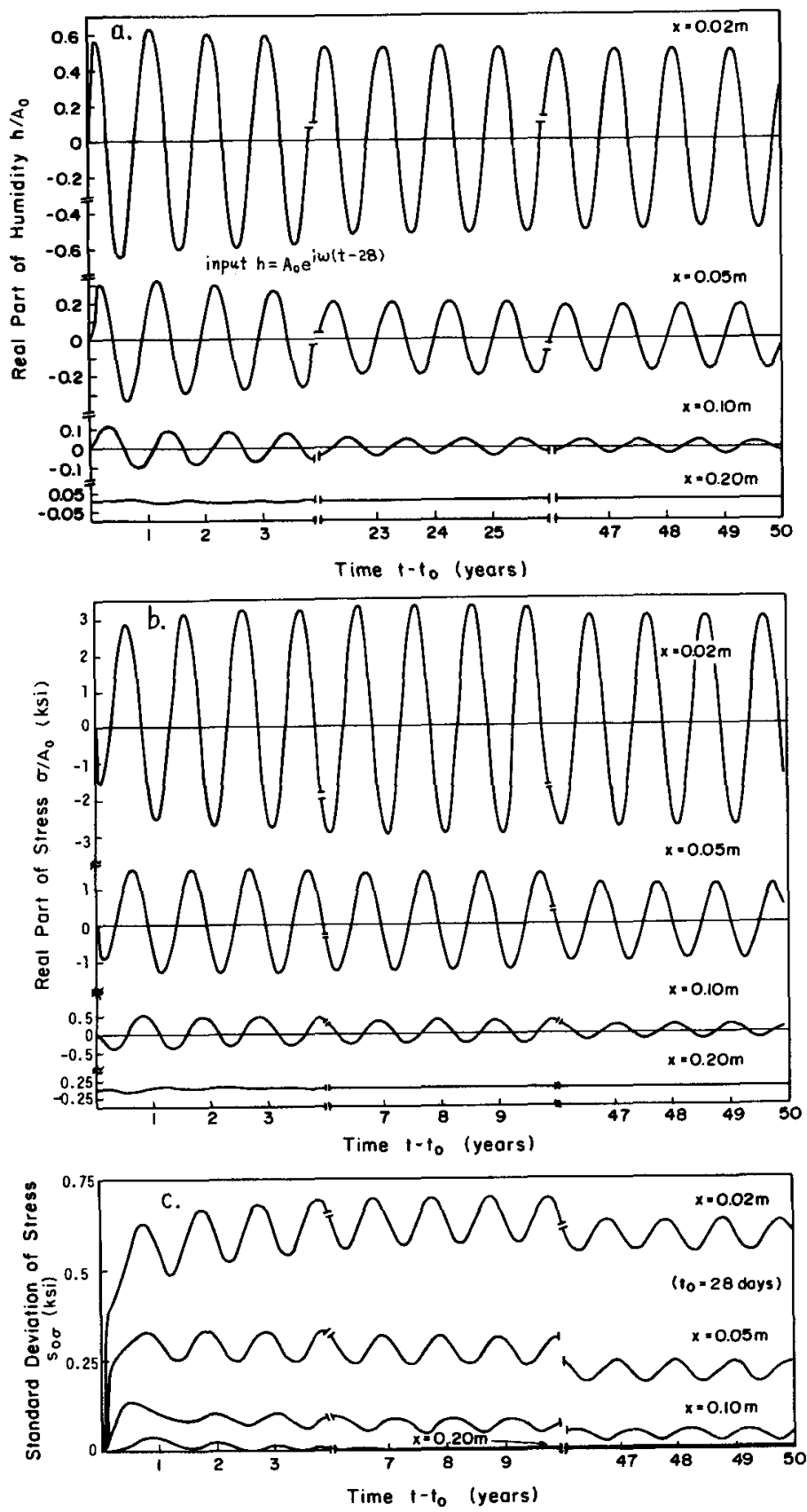

FIG. 3.-Time Variations of: (a) Pore Humidity; (b) of Stress; and (c) of Standard Deviation of Stress, at Various Depths below Surface

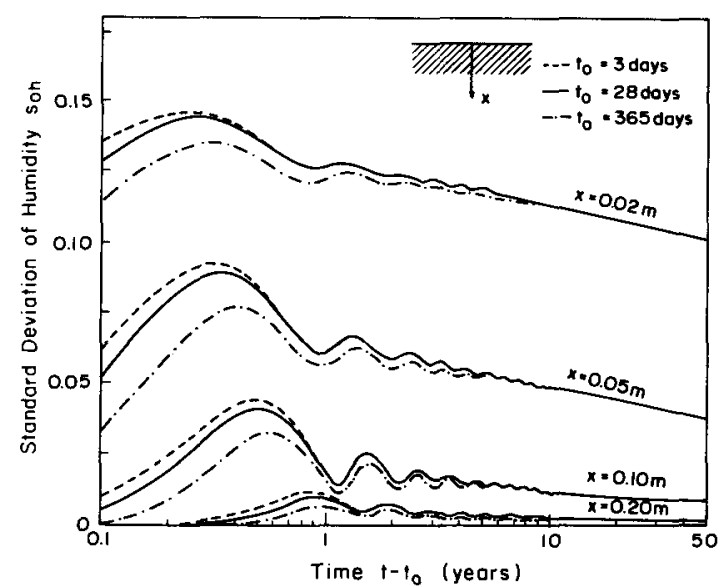

FIG. 4.-Variation of Standard Deviation of Pore Humidity at Various Depths, in Logarithmic Time Scale
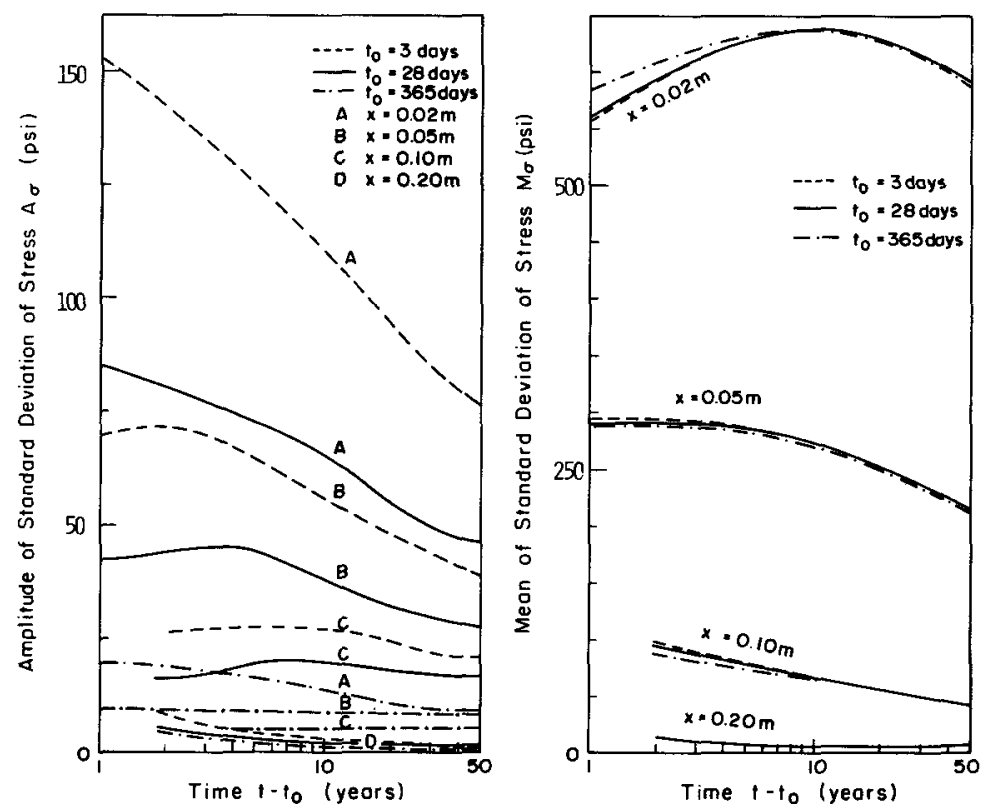

FIG. 5.-Time Varlations of Mean and Amplitude of Oscillations for Standard Devlation of Stress

does not attain a stationary value within a 50-yr lifetime, due to both the small value of diffusivity and its decline with age.

The histories of stress (its real part) at various depths are plotted in Fig. $3(b)$. Due to the hardening of concrete with age, i.e., a decrease in 
creep compliance with the age at loading, the stress amplitude keeps increasing at first (for the first few years), and then it declines as the stress relaxation and the decrease of diffusivity prevail.

The time variation of the standard derivation of stress, $s_{0 \sigma}$, is shown in Fig. $3(c)$. It is noteworthy again that $s_{0_{\sigma}}$ oscillates, except for the first few months. The amplitude, $A_{s}$, and the average $M_{s}$ of $s_{0 \sigma}$ [determined in a manner explained by Fig. $1(d)]$, are plotted in Fig. 5 . The values of $A_{s}$ increase with the decrease of $x$, or of $t_{0}$. When the depth, $x$, increases, $M_{s}$ rapidly decreases, and the curves of $M_{s}$ for different values of $t_{0}$ are getting closer. (It may be noted that the values of $s_{0 \sigma}$ for the thick-wall cylinder solved in Ref. 16 oscillate, too; the graphs in Ref. 16 do not show it, unfortunately, because they were plotted from calculated points that were spaced too far apart.)

The stresses produced by humidity fluctuations are rather high and far exceed the tensile strength. Thus, the present solution, based on assuming linearity, is applicable only when the permanent loads or prestress, or both, produce superimposed compressive stresses that reduce the combined tensile stress peaks below the strength limit. Otherwise, tensile strain-softening would have to be taken into account, which would make the problem nonlinear.

\section{Conclusions}

1. The age-dependence of material properties causes the frequency response functions of pore humidity and stress, as well as their standard deviations, to depend on time, both on the current time and on the age at the start of drying. The equations governing the frequency response functions are of integral or differential type in time, rather than algebraic

2. The time variations of the standard deviation of pore humidity and of stress exhibit oscillations about a drifting mean.

3. For the usual values of drying diffusivity, the solution is nonstationary for at least a 50-yr lifetime, and for environmental fluctuations whose period does not exceed $1 \mathrm{yr}$, the fluctuations are not felt at depths over $20 \mathrm{~cm}$ (for a concrete of typical diffusivity).

4. The use of the spectral method, instead of the impulse response method, is more efficient numerically since the spectral densities of input and of response are related algebraically.

5. The present linear analysis is applicable only when the permanent loads or prestress, or both, reduce the stress peaks below the tensile strength limit.

\section{ACKNOWLEDGMENT}

Partial financial support under U.S. National Science Foundation Grant No. CEE-8303148 is gratefully acknowledged. Mary Hill is thanked for her expert secretarial assistance.

\section{Appendix l.-Stress Fluctuation at Surface}

Assuming the pore humidity at the surface, $x=0$, to be equal to the environmental humidity, we can express the stress, $\sigma_{y}$, at the surface analytically if the moduli $E_{\mu}\left(t^{\prime}\right)$ in Eq. 24 for $R\left(t, t^{\prime}\right)$ are expressed also as a Dirichlet series (5), i.e.

$E_{\mu}\left(t^{\prime}\right)=E_{0 \mu}+\sum_{j=1}^{n} E_{j \mu} e^{-t^{\prime} / \tau_{j}} ; \quad E_{\infty}\left(t^{\prime}\right)=E_{0 \infty}+\sum_{j=1}^{n} E_{j \infty} e^{-t^{\prime} / \tau_{j}} \ldots \ldots \ldots$

in which $E_{j \mu}, \ldots E_{0 \infty}=$ constants (whose determination from test data is described in Ref. 5). The values of $\tau_{j}(j=1,2 \ldots)$ may be chosen to be the same as the values of $\tau_{\mu}(\mu=1,2 \ldots)$. Substituting $h\left(0, t^{\prime}\right)$ $s_{0 h} e^{i \omega\left(t^{\prime}-t_{0}\right)}$ and Eq. 24 with Eq. 25 into Eq. 17, in which $\nu=$ const., $\kappa_{\text {sh }}$ $=$ const., we may easily integrate term by term and obtain

$\sigma_{y}(0, t)=\left[\sigma_{1}+\sigma_{2} e^{i \omega\left(t-t_{0}\right)}+\sigma_{3} e^{-t / \tau_{\mu}}+\sigma_{4} e^{i \omega\left(t-t_{0}\right)} e^{-t / \tau_{j}}\right] s_{0 h} \ldots \ldots \ldots$

in which $\sigma_{1}, \sigma_{2}, \sigma_{3}, \sigma_{4}$ are complex constants:

$\sigma_{1}=\sum_{j=1}^{n} \hat{E}_{j \infty} e^{-t_{0} / \tau_{j}} \frac{\kappa_{s h}}{(1-\nu)\left(i \omega \tau_{j}-1\right)} ; \quad \sigma_{2}=-\sum_{\mu-1}^{m} E_{0 \mu} \frac{\kappa_{s h}\left(1+2 i \omega \tau_{\mu}\right)}{(1-v)\left(1+i \omega \tau_{\mu}\right)} ;$

$\sigma_{3}=\sum_{\mu=1}^{m}\left(-E_{0 \mu} e^{t_{0} / \tau_{\mu}} \frac{1}{1+i \omega \tau_{\mu}}\right.$

$\left.+\sum_{j=1}^{n} \hat{E}_{j \mu} e^{\left[\left(1 / \tau_{\mu}\right)-\left(1 / \tau_{j}\right)\right] t_{0}} \frac{\tau_{\mu}-\tau_{j}}{i \omega \tau_{\mu} \tau_{j}-\tau_{\mu}+\tau_{j}}\right) \frac{\kappa_{s h}}{1-\nu} ;$

$\sigma_{4}=-\sum_{j=1}^{n}\left(\sum_{\mu=1}^{m} \hat{E}_{j \mu} \frac{i \omega \tau_{\mu} \tau_{j}}{i \omega \tau_{\mu} \tau_{j}-\tau_{\mu}+\tau_{j}}+\hat{E}_{j \infty} \frac{i \omega \tau_{j}}{i \omega \tau_{j}-1}\right) \frac{\kappa_{s h}}{1-v} \ldots \ldots \ldots$

The limiting stationary response for $t \rightarrow \infty$ is $\sigma_{y}=\sigma_{1}+\sigma_{2} e^{i \omega /\left(t-t_{0}\right)} s_{0 h}$. The variance for $t \rightarrow \infty$ is $s_{0 \sigma}^{2}=\sigma_{y} \sigma_{y}^{*}+\sigma_{1} \sigma_{1}^{*}+\sigma_{2} \sigma_{2}^{*}+\sigma_{1}^{*} \sigma_{2} e^{i \omega\left(t-t_{0}\right)}+$ The variance for $t \rightarrow \infty$ is $s_{0 \sigma}^{2}=\sigma_{y} \sigma_{y}^{*}+\sigma_{1} \sigma_{1}^{*} \sigma_{2} \sigma_{2}$. $\sigma_{1} \sigma_{2}^{*} e^{i \omega\left(t-t_{0}\right)}$. We see that this is a fluctuating function is the variance for any $t$. The reason that the variance of the rensemfluctuates with age is the fact that statistics is not applied to the ensemble of responses at various ages, $t$, but to the ensemble of the random the same age, environment.

\section{Appendix II.-References}

.Theory of Creep and Shrinkage in Concrete Structures: A Préci, Zechanics Today, Vol. 2, Pergamon Press, Précis of Recent Developments,

New York, N.Y., 1975, pp. 1-93.

2. Bažant, Z. P., "Mathematical Models for Creep and Y. P. Bažant and F. H. Chpt. 7 in Creep and Shrinkage in Concrete Structures, $Z$.

Wittmann, eds., J. Wiley, London, 1982, pp. 163-256.

3. Bažant, Z. P., "Response of Aging Linear Systems to Random Input," Concrete and Geomaterials Report No. 82-12/665r, Northwestern University, Evanston, III., Dec., 1982.

Problems in Prediction of Creep and Shrinkage 4. Bažant, Z. P., "Probabilistic Problems in Prediction of Creep anderence on AppliEffects in Structures," Proceedings, 4th International Conference on Applorcation of Statistics and Probability in

ence, Italy, June, 1983, pp. 325-356.

5. Bažant, Z. P., and Asghari, A., "Computation of Age-Dependent Re Spectra," Cement $\mathcal{E}$ Concrete Research, Vol. 4, Water Diffucion in Nonsaturated 
Concrete," Materials and Structures, Paris, France, Vol. 5, 1972, pp. 3-20.

7. Bažant, Z. P., and Wu, S. T., "Creep and Shrinkage Law for Concrete at Variable Humidity," Journal of the Engineering Mechanics Division, ASCE, Vol 100 , No. EM6, Dec, 1974, pp. 1183-1207.

8. Bažant, Z. P., and Wu, S. T., "Rate-Type Creep Law of Aging Concrete Based on Maxwell Chain," Materials and Structures, Paris, France, Vol. 7, No. 37, 1974, pp. 45-60.

9. Bažant, Z. P., Carreira, D., and Walser, A., "Creep and Shrinkage in Reactor Containment Shells," Journal of the Structural Division, ASCE, Vol. 101, 1975, pp. 1997-2004.

10. Carslaw, H. S., and Jaeger, J. C., Conduction of Heat in Solids, 2nd ed., Clarendon Press, Oxford, 1959

11. Crandall, S. H., Random Vibration in Mechanical Systems, Academic Press, New York, N.Y., 1963

12. "Finite Element Analysis of Reinforced Concrete," State-of-the-Art Report by ASCE Structural Division Task Committee, chaired by A. Nilson, ASCE, New York, 1982, pp. 309-400.

13. Heller, R. A.," "Temperature Response of an Infinitely Thick Slab to Random Surface Temperatures," Mechanics Research Communication, Vol. 3, 1976, pp. 379-385.

14. Heller, R. A., "Thermal Stress as a Narrow-Band Random Load," Journal of the Engineering Mechanics Division, ASCE, Vol. 102, No. EM5, Oct., 1976, pp. 787-805.

15. Papoulis, A., Probability, Random Variables and Stochastic Processes, McGrawHill, New York, N.Y., 1965.

16. Tsubaki, T., and Bažant, Z. P., "Random Shrinkage Stresses in Aging Viscoelastic Vessel," Journal of the Engineering Mechanics Division, ASCE, Vol. 108 No. EM3, June, 1982, pp. 527-545.

17. Wierig, H. J., "Die Wasserdampfdurchlässigheit von Zementmörtel und Beton," Zement-Kalk-Gips, Nov., 1965, No. 9, pp. 47-482.

18. Zhu, B. F., Wang, T. S., et al., "Temperature Stresses and Temperature Control in Hydraulic Concrete Structures," Water Power Press, Beijing, China 1976 (in Chinese)

\section{ApPendix III.-Notation}

The following symbols are used in this paper:

$A=$ amplitude of environmental humidity;

$C=$ drying diffusivity in concrete;

$E=$ expectation;

$E, E_{c}=$ creep operator and Young's modulus of concrete;

$E_{\mu}\left(t^{\prime}\right)=$ relaxation moduli of Maxwell chain at time $t^{\prime}(\mu=1,2$, ...m);

$h=$ complex variable whose real part is pore humidity in pores of concrete;

$R\left(t, t^{\prime}\right)=$ relaxation function of concrete;

$s_{0 h}=$ standard deviation of amplitude of environmental humidity;

$s_{0 h}=$ standard deviation of humidity;

$s_{0 \sigma}=$ standard deviation of stress:

$t=$ current time;

$t^{\prime}=$ age of concrete

$x=$ spatial coordinate for points in structure

$Y=$ frequency response function:

$\kappa_{s h}=$ shrinkage coefficient;

$\begin{aligned} v & =\text { Poisson's ratio of concrete; } \\ \sigma_{x}, \sigma_{y}, \sigma_{z} & =\text { normal stresses; } \\ \epsilon_{x}, \epsilon_{y}, \epsilon_{z} & =\text { normal strains; } \\ \epsilon_{s h}= & \text { shrinkage strain; and } \\ \omega= & \text { circular frequency of component of environmental hu- } \\ & \text { midity. }\end{aligned}$

